\title{
Risiken des Klimawandels: Wie kann man mit den vielfältigen Unsicherheiten bei Risikobewertung und Anpassung an den Klimawandel umgehen?
}

\section{Petra Döll}

\section{Zusammenfassung}

Um die vielfältigen Risiken des Klimawandels zu verringern, ist es notwendig, diese in lokalen partizipativen Prozessen mit Stakeholdern und Wissenschaftler*innen $\mathrm{zu}$ analysieren und $\mathrm{zu}$ bewerten und Strategien zur Risikoverringerung entwickeln. Dabei spielen verschiedene Arten von Unsicherheiten eine wichtige Rolle und sollten explizit adressiert werden. Anhand einer Unsicherheitsklassifikation werden die für solche partizipativen Prozesse relevanten Unsicherheiten (epistemische, ontologische und linguistische Unsicherheiten sowie Uneindeutigkeit) diskutiert, und es werden Methoden zum Umgang mit diesen Unsicherheiten vorgestellt.

\section{$1 \quad$ Einleitung}

Alle Gesellschaften sind gezwungen, mit den vielfältigen Risiken des menschgemachten Klimawandels (KW) umzugehen und den Klimawandel durch reduzierte Treibhausgasemissionen zu begrenzen und sich an das geänderte Klima anzupassen. KW-Risiken werden als potenzielle zukünftige Auswirkungen gefährlicher Ereignisse oder Trends, die vom KW verursacht werden, definiert (IPCC 2014). Es wird mittlerweile als sinnvoll angesehen, von KW-Risiken statt von $\mathrm{KW}$ Auswirkungen oder -Folgen zu sprechen. Der Begriff Risiko spiegelt zum einen wider, dass es unsicher ist, wie sich der Klimawandel und seine Folgen entwickeln

P. Döll (ه)

Institut für Physische Geographie der Goethe-Universität Frankfurt am Main, Frankfurt am Main, Deutschland

E-Mail: p.doell@em.uni-frankfurt.de 
werden. Zum anderen haben Politik und Wirtschaft Erfahrung im Management vielfältiger Risiken, die in das $\mathrm{KW}$-Risikomanagement eingebracht werden kann. KW-Risiken entstehen durch das Zusammenspiel von 1) KW-Gefahren (oder auch „Gefährdungen“), d. h. gefährlichen Ereignissen oder Trends in der Umwelt, die durch den KW verursacht werden, mit 2) Ausgesetztsein (Exposition) und 3) Verwundbarkeit (Vulnerabilität) (IPCC 2014). Beispielsweise kann das Risiko für Menschen, in Zukunft aufgrund des KW an verringerten Grundwasserressourcen zu leiden, durch die mögliche Stärke der Abnahme der Grundwasserressourcen (Gefahr), die Anzahl der Menschen, die mit Grundwasser versorgt werden (Exposition) und der Abhängigkeit von Grundwasser als Wasserquelle (Verwundbarkeit) beschrieben werden (Döll 2009).

Der Weltklimarat (Intergovernmental Panel on Climate Change IPCC) schlägt ein iteratives und partizipatives KW-Risikomanagement vor (IPCC 2014). Aufgrund von Ausmaß, Komplexität, Unsicherheit und Uneindeutigkeit (Ambiguität) der KW-Risiken muss die KW-Risikobewertung ebenso wie die Entwicklung von Maßnahmen kooperativ durch eine breite Gruppe von Wissenschaftler*innen und Stakeholdern (Betroffene und Entscheidungsträger*innen) geschehen (Mimura et al. 2014). Eine Herausforderung ist, für ein solches KW-Risikomanagement transdisziplinäre partizipative Prozesse auf lokaler bis nationaler Ebene zu gestalten, die eine effektive und sinnvolle Integration des Wissens von multisektoralen Stakeholdern und multidisziplinären Wissenschaftler*innen ermöglichen, ebenso wie die Berücksichtigung von unterschiedlichen Werten und legitimen Problemsichten der Stakeholder (Renn et al. 2011). Während bereits vielfältige Erfahrungen mit partizipativen Prozessen gemacht worden sind, z. B. im Problemfeld nachhaltiges Land- und Wassermanagement, müssen die wissenschaftlichen Grundlagen zur Gestaltung von partizipativen Prozessen noch weiterentwickelt werden (Lang et al. 2012; Scholz und Steiner 2015).

KW-Risikomanagement beinhaltet die Bewertung von KW-Risiken sowie die Identifizierung von Maßnahmen zur Risikoverringerung. Beide Schritte werden durch vielfältige Unsicherheiten erschwert. Im Folgenden werden Unsicherheiten, die in partizipativen KW-Risikomanagementprozessen (PRMP) relevant sind, basierend auf einer neu entwickelten Unsicherheitsklassifikation vorgestellt (Döll und Romero-Lankao 2017). Danach werden Vorschläge gemacht, wie unterschiedliche Unsicherheiten in PRMP explizit behandelt werden können. 


\section{Unsicherheiten im KW-Risikomanagement}

Der Begriff „Unsicherheit“ hat für Menschen in verschiedenen Kontexten eine unterschiedliche Bedeutung. Döll und Romero-Lankao (2017) haben daher eine Unsicherheitsklassifikation vorgeschlagen, mit der verschiedene Arten von Unsicherheiten in PRMP umfassend behandelt werden können. Sie verbindet Elemente und Ideen von Walker et al. (2003), Ascough et al. (2008), Kwakkel et al. (2010) und Bijlsma et al. (2011). Unsicherheit kann als jegliche Abweichung vom unerreichbaren Ideal eines umfassenden Wissens und Verständnisses, das alle teilen, definiert werden (Walker et al. 2003, erweitert). Mithilfe der Unsicherheitsklassifikation soll es den Teilnehmer*innen von PRMP ermöglicht werden, alle im Rahmen von PRMP auftretenden Unsicherheiten zu erkennen und explizit zu machen und dann geeignet mit diesen umzugehen.

Jede Unsicherheit wird entsprechend ihrer Position, ihrer Art und ihres Grades beschrieben (Abb. 1). So könnten das Ausmaß zukünftiger Treibhausgasemissionen oder der Begriff „Nachhaltigkeit“ als Positionen von Unsicherheiten, die für den spezifischen PRMP von Bedeutung sind, identifiziert werden. Für jede Position wird dann zunächst festgestellt, welche Art von Unsicherheit die Position betrifft. In PRMP sind epistemische, ontologische und linguistische Unsicherheiten ebenso relevant wie die Uneindeutigkeit (Abb. 1). Uneindeutigkeit wird hier als eine Art von Unsicherheit betrachtet, da durch sie insbesondere eine eindeutige Risikoanalyse und -bewertung unmöglich gemacht wird, die von allen geteilt wird und ein für alle sicheres Wissen widerspiegelt. Schließlich wird der Grad der Unsicherheit bestimmt (Abb. 1). Eine so strukturierte Erfassung von Unsicherheiten erleichtert den Umgang mit Unsicherheiten in PRMP. Im Folgenden werden die unterschiedlichen Arten von Unsicherheiten erläutert und für das KW-Risikomanagement wichtige Positionen beschrieben, bei denen solche Unsicherheiten auftreten.

\subsection{Epistemische Unsicherheiten}

Unvollständiges Wissen führt zu epistemischer Unsicherheit. Substanzielle epistemische Unsicherheit wird durch unvollständiges Wissen über das Problemfeld an sich verursacht, d. h. durch begrenztes Systemwissen (Wie funktioniert das problemrelevante System?), Zielwissen (Welche Ziele haben die beteiligten Akteure?) und Transformationswissen (Wie kann das Ziel erreicht werden?). Im Rahmen partizipativer Prozesse gibt es darüber hinaus nur begrenztes Wissen darüber, wie die am Prozess Beteiligten agieren werden und wie der Prozess durch 


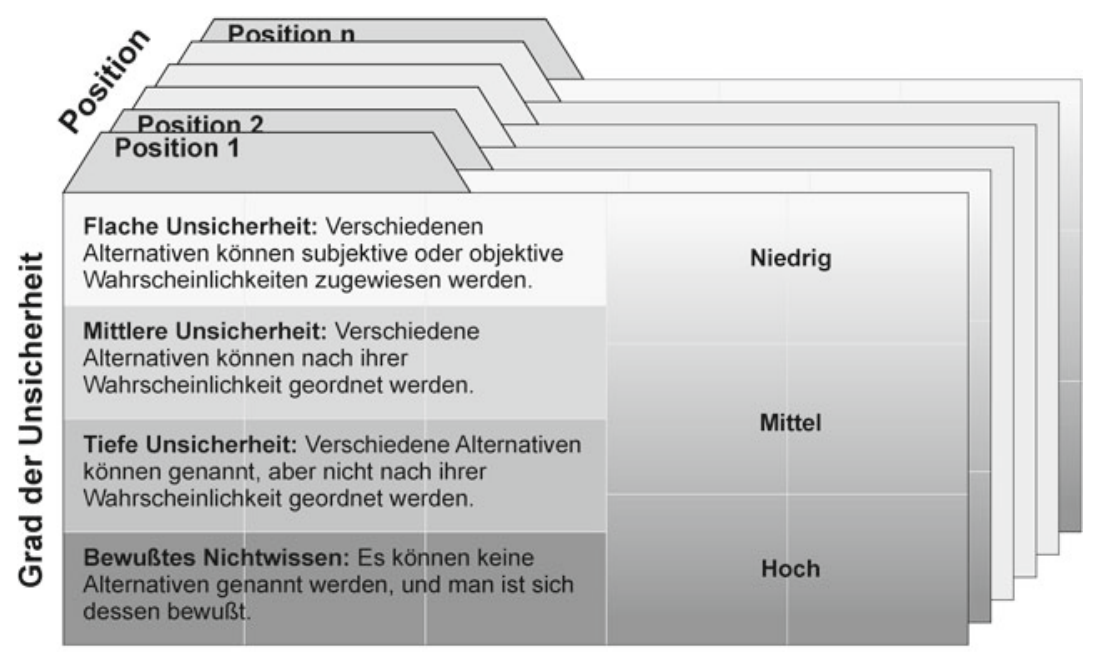

\section{Art der Unsicherheit}

Epistemisch
aufgrund begrenzten Wissens
Substantiell $\quad$ Part. Prozess
über System, über Handlungen
$\begin{array}{cc}\text { Ziele und Wege } & \text { der Teilnehmer } \\ \text { zum Ziel } & \text { oder externe } \\ & \text { Einflüsse }\end{array}$

$\begin{array}{ccc}\begin{array}{c}\text { Ontologisch } \\ \text { aufgrund }\end{array} & \begin{array}{c}\text { Uneindeutigkeit Linguistisch } \\ \text { aufgrund aufgrund der }\end{array} \\ \text { inhärenter } & \text { unterschiedlicher Sprache } \\ \text { Variabilität } & \begin{array}{c}\text { legitimer } \\ \text { Perspektiven und } \\ \text { Rahmung }\end{array}\end{array}$

Abb. 1 Beschreibung einer Unsicherheit nach Position, Art und Grad. (Nach Döll und Romero-Lankao 2017). Die Position bezieht sich auf die Komponente des untersuchten Problemfelds, bei der die Unsicherheit auftritt, z. B. die zukünftigen Treibhausgasemissionen. Bei epistemischen oder ontologischen Unsicherheiten beschreibt der Grad der Unsicherheit, wie genau das Eintreten möglicher alternative Zustände prognostiziert werden kann, die sich aufgrund von menschlichen Handlungen oder natürlichen Prozesse einstellen können (z. B. die Menge der Treibhausgasemissionen)

externe Ereignisse, z. B. übergeordnete Politikentscheidungen, beeinflusst werden wird. Im Folgenden werden substanzielle epistemische Unsicherheiten, die für ein KW-Risikomanagement wichtig sind, beschrieben.

Für die Quantifizierung der KW-Gefahren ist es problematisch, dass die zukünftigen Treibhausgasemissionen, die die KW-Gefahren beeinflussen, und die sozioökonomischen Bedingungen, die für Exposition und Vulnerabilität verantwortlich sind, nicht prognostiziert werden können, insbesondere da beide von vielen Entscheidungen vieler Menschen abhängen. Wir können mögliche zukünftige Entwicklungspfade nennen, aber keine gesicherten Aussagen darüber machen, 
welche Pfade jeweils wahrscheinlicher sind als andere. Diese Art von epistemischer Unsicherheit kann als ,tiefe“ Unsicherheit bezeichnet werden (Abb. 1; Kwakkel et al. 2010; Döll und Romero-Lankao 2017). Aufgrund dieser „tiefen“ Unsicherheit wird bei der Bewertung von KW-Risiken statt mit probabilistischen Vorhersagen (,ein bestimmtes Ereignis wird mit einer Wahrscheinlichkeit von x auftreten“) mit Szenarien zukünftiger Treibhausgase und zukünftiger sozioökonomischer Bedingungen gearbeitet. Szenarien sind alternative Zukünfte, die plausibel und konsistent sind und die nicht durch Eintrittswahrscheinlichkeiten beschrieben werden können.

Des Weiteren ist die Quantifizierung zukünftiger KW-Gefahren von Unsicherheiten bezüglich der Klimaänderungen und Klimaänderungsfolgen, die sich bei den jeweiligen Treibhausgasszenarien oder globalen Erwärmungen einstellen werden, betroffen. Vier Szenarien zukünftiger Treibhausgasemissionen wurden von globalen Klimamodellen in Klimaänderung, d. h. Klimaszenarien, ,übersetzt" (IPCC 2014). In Klimaszenarien wird die zukünftige Entwicklung von Temperatur, Niederschlag, Wind und anderen Klimavariablen in der Atmosphäre quantifiziert, die stattfinden könnte, wenn ein bestimmtes Emissionsszenario wahr würde (IPCC 2014). Aufgrund der Komplexität des Klimasystems, das nicht nur die Atmosphäre, sondern auch den Ozean und die Landflächen umfasst, berechnen unterschiedliche Klimamodelle meist deutlich unterschiedliche Klimaszenarien für ein gegebenes Emissionsszenario, wobei die Unterschiede und damit die Unsicherheiten beim Niederschlag deutlich größer sind als bei der Temperatur. Diese Art von Unsicherheit kann als „flach“ bis ,mittel“" bezeichnet werden (Kwakkel et al. 2010; Döll und Romero-Lankao 2017). Eine Unsicherheit wird als flach definiert, falls den Alternativen eine Wahrscheinlichkeit zugewiesen werden kann, und als mittel, falls die Alternativen nach ihrer Wahrscheinlichkeit geordnet werden können (Abb. 1). Aussagen zur Unsicherheit von Klimaszenarien können getroffen werden, da eine Vielzahl von Klimamodellen (20-40) die vier Emissionsszenarien durchgerechnet haben. Betrachtet man die Berechnungsergebnisse der einzelnen Klimamodelle als gleich wahrscheinlich und nimmt an, dass diese den gesamten Wahrscheinlichkeitsraum abdecken, können Wahrscheinlichkeiten der zukünftigen Klimaänderungen für das jeweilige Emissionsszenario berechnet werden. Diese Annahmen treffen aber nur in erster Näherung zu (Döll et al. 2015), weswegen die Unsicherheit der Klimaszenarien unter der Bedingung, dass das in den Modellen berücksichtigte Emissionsszenario eintreten wird, flach bis mittel ist. Um nun die Gefahren des Klimawandels in verschiedenen Sektoren wie Landwirtschaft oder Wasserversorgung abzuschätzen, werden die Klimaszenarien als Eingabegrößen sektoraler Impaktmodelle, z. B. von landwirtschaftlichen Ertragsmodellen oder hydrologischen Modellen, verwendet. Dabei ist 
es üblich, die globalen Klimaszenarien mithilfe lokaler Klimabeobachtungen zu korrigieren (durch Downscaling und/oder Bias-Korrektur). In der Modellkaskade pflanzen sich die substanziellen epistemischen Unsicherheiten fort. Durch die sektorale Modellierung und die Korrekturen werden wiederum flache bis mittlere Unsicherheiten eingeführt, sodass für die quantitative Abschätzung von Gefahren des Klimawandels, für ein gegebenes Emissionsszenario oder eine bestimme globale Erwärmung, insgesamt wohl eine mittlere Unsicherheit vorliegt, d. $h$. alternative Gefahrenstärken können grob nach ihrer Wahrscheinlichkeit geordnet werden (Abb. 1). Weitere Positionen von epistemischen Unsicherheiten für die Quantifizierung von KW-Gefahren sind in Tab. 3 in Döll und Romero-Lankao (2017) gelistet.

Die substanziellen epistemischen Unsicherheiten, die bei Abschätzung von Exposition und Verwundbarkeit auftreten (siehe Tab. 3 in Döll und RomeroLankao 2017), sind keineswegs kleiner als die der KW-Gefahren. So ist schon oft die Beschreibung der heutigen Verwundbarkeit gegenüber dem KW unsicher (flache bis mittlere Unsicherheit je nach Kontext), während die zukünftige Entwicklung der Verwundbarkeit durch eine tiefe Unsicherheit geprägt ist. Schließlich gibt es verschiedene Positionen von Unsicherheiten bei der Identifizierung von Klimaschutz- und Klimaanpassungsmaßnahmen (siehe Tab. 4 in Döll und Romero-Lankao 2017). Von großer Bedeutung für das KW-Risikomanagement ist, dass das Transformationswissen (z. B. mit welchen Mitteln eine Verhaltensänderung erreicht werden kann) sehr oft geringer ist als das Systemwissen. Daher sind die Unsicherheiten bezüglich geeigneter Maßnahmen für Klimaschutz und die Anpassung an den Klimawandel sehr oft größer als die bezüglich der Risikoanalyse.

\subsection{Ontologische Unsicherheiten}

Ontologische Unsicherheit tritt aufgrund der inhärenten Variabilität natürlicher und menschlicher Systeme auf. Sie wird auch zufällige oder stochastische Unsicherheit genannt. Für ein KW-Risikomanagement besonders relevant sind ontologische Unsicherheiten, die aufgrund der stochastischen Natur des Wetters auftreten. Diese macht es unmöglich, das Auftreten eines Wetterereignisses oder eines vom Wetter ausgelösten Ereignisses (z. B. Hochwasser) vorherzusagen. Ist die stochastische Beschreibung des Phänomens aufgrund von langjährigen Beobachtungszeitreihen gut bekannt, d. h. nicht unsicher, liegt keine epistemische Unsicherheit vor. Durch den Klimawandel ist jedoch eine neue epistemische Unsicherheit bei der stochastischen Beschreibung des Wetters hinzugekommen, da 
Wahrscheinlichkeiten (z. B. für das Auftreten bestimmter Hochwasserereignisse) nicht mehr aus Beobachtungszeitreihen abgeleitet werden können (,,Stationarity is dead“, Milly et al. 2008). Eine weitere ontologische Unsicherheit in PRMP ergibt sich aufgrund der zufälligen Zusammensetzung der Teilnehmer*innen.

\subsection{Uneindeutigkeit}

Uneindeutigkeit ist eine Art Unsicherheit, die dadurch auftritt, dass Personen oder Organisationen unterschiedliche legitime Sichtweisen auf das betrachtete Problem sowie unterschiedliche Bezugsrahmen haben, sodass sie (z. B. bei der Analyse und Bewertung eines KW-Risikos) auch dann nicht übereinstimmen könnten, wenn keinerlei andere Unsicherheiten vorhanden wären (Renn 2008). So können beispielsweise unterschiedliche Auffassungen über die Wichtigkeit bestimmter Systemkomponenten existieren und $\mathrm{zu}$ unterschiedlichen Interpretationen von Daten führen. Uneindeutigkeit kann auch zu einer unterschiedlichen Bewertung der Bedeutung von epistemischen Unsicherheiten führen. Zum einen kann das Risiko, dass eine bestimmte negative Auswirkung mit einer Wahrscheinlichkeit von $0,01 \%$ auftreten wird, von manchen als gering und von anderen als hoch eingeschätzt werden, sodass erstere risikoreduzierende Maßnahmen ablehnen und letztere diese befürworten. Zum anderen kann epistemische Unsicherheit über einen Sachverhalt bei Menschen mit geringem Vertrauen in wissenschaftliche Erkenntnisse dazu führen, dass sie diese Erkenntnisse für ihre Risikobewertung überhaupt nicht berücksichtigen. Uneindeutigkeit wird durch unterschiedliche Wertesysteme, Erfahrungen, Erwartungen und Wissensformen verursacht (Renn 2008; Kwakkel et al. 2010; Renn et al. 2011). Uneindeutigkeit bleibt auch dann bestehen, wenn die Unsicherheit bezüglich des Zielwissens der Akteure im Laufe des PRMP verringert worden ist, indem die Teilnehmer*innen sich über ihre Ziele ausgetauscht haben. Uneindeutigkeit ist auf einem niedrigen Niveau, wenn (fast) alle ein gemeinsames Problemverständnis haben. Erfahrungen beim partizipativen Hochwassermanagement (ohne Betrachtung des Klimawandels) haben gezeigt, dass Uneindeutigkeit für die Entscheidungsfindung in partizipativen Prozessen bedeutender sein kann als epistemische und ontologische Unsicherheiten (Van den Hoek et al. 2014). 


\subsection{Linguistische Unsicherheiten}

Linguistische oder sprachliche Unsicherheiten treten auf, weil unsere Sprache vage, mehrdeutig, nicht exakt und kontext-abhängig ist (Ascough et al. 2008). Falls die Teilnehmer*innen des PRMP nicht dieselbe Muttersprache haben, sind linguistische Unsicherheiten besonders groß.

\section{Umgang mit Unsicherheiten in PRMP}

Die Durchführung von PRMP ist die Methode der Wahl, um mit den vielfältigen und verknüpften Unsicherheiten des $\mathrm{KW}$ umzugehen. Zum einen können Stakeholder aus multiplen Sektoren und Wissenschaftler*innen aus multiplen Disziplinen ihr diverses System-, Ziel- und Transformationswissen integrieren und so die epistemische Unsicherheit verringern. Zum anderen kann Uneindeutigkeit durch geeignete partizipative Methoden transparent gemacht werden, und unterschiedliche Problemperspektiven können bis zu einem gewissen Grad harmonisiert werden.

PRMP bestehen aus verschiedenen Phasen, z. B. einer Vorbereitungsphase und einer Hauptphase, die wiederum in die Phasen Einleitung, Risikoidentifizierung, Risikoanalyse, Risikobewertung und Entwicklung einer Risikomanagementstrategie gegliedert werden kann (Döll und Romero-Lankao 2017). In jeder Phase sollten die relevanten Unsicherheiten explizit adressiert und behandelt werden. Dadurch können alle Arten von Unsicherheiten mit Ausnahme der ontologischen verringert werden. Nicht weiter reduzierbare Unsicherheiten sollten transparent dargestellt und vor allem bei der Risikobewertung und der Strategieentwicklung berücksichtigt werden. Döll und Romero-Lankao (2017) geben konkrete Hinweise, wie dies in den unterschiedlichen Phasen des PRMP geschehen kann, nachdem sie die in der jeweiligen Phase wichtigen Unsicherheiten genannt haben.

Beispielsweise kann linguistische Unsicherheit durch präzise Begriffsdefinition verringert werden, so wie dies durch die kalibrierte Unsicherheitssprache des IPCC erreicht werden soll (Mastrandrea et al. 2011, deren Tab. 11). Carey und Burgman (2008) stellten fest, dass eine explizite Behandlung der linguistischen Unsicherheit die Übereinstimmung unter Workshop-Teilnehmer*innen bezüglich der wichtigsten Risiken erhöht hatte.

Uneindeutigkeit aufgrund unterschiedlicher Problemwahrnehmungen der Teilnehmer*innen des PRMP kann durch individuelle Wahrnehmungsgraphen transparent und durch die Entwicklung gemeinsamer Wahrnehmungsgraphen reduziert werden (Titz und Döll 2009; Döll et al. 2013; Düspohl und Döll 2016). Zudem 
kann durch die Wahrnehmungsgraphen die epistemische Unsicherheit insbesondere hinsichtlich des Ziel- und Transformationswissens verringert werden.

Die Identifizierung geeigneter Klimaschutz- und Anpassungsmaßnahmen wird stark durch die tiefe epistemische Unsicherheit der zukünftigen sozioökonomischen Entwicklungen beeinträchtigt. Hier eignet sich die gemeinsame Erstellung von qualitativen Zukunftsszenarien, mit alternativen sozioökonomischen Entwicklungen, durch die Stakeholder im PRMP, um trotz der tiefen epistemischen Unsicherheit optimale Handlungsstrategien zu entwickeln (Döll et al. 2013; Düspohl und Döll 2016). Insbesondere können robuste Strategien, die in vielen plausiblen Zukünften positive Auswirkungen erwarten lassen, identifiziert werden. Van Notten (2006) gibt einen Überblick über verschiedenen Arten von Szenarien. Zur Identifizierung von Klimaschutzstrategien können normativen Szenarien erstellt werden, in denen alternative Wege zum Erreichen eines Ziels beschrieben werden. Z. B. kann gemeinsam erarbeitet werden, wie ein bestimmtes Emissionsreduktionsziel in einer administrativen Einheit erreicht werden kann, angesichts unsicherer externer (z. B. Fortbestand einer Subventionierung) oder interner (z. B. Energiebedarfsentwicklung) Faktoren. Dazu wird eine kleine Anzahl von Szenarien entworfen, bei denen für diese Faktoren jeweils alternative Annahmen getroffen werden und erzählt wird, wie bei diesen Annahmen das Ziel erreicht werden kann (Düspohl und Döll 2016). Explorative (deskriptive) Szenarien beschreiben alternative Zukünfte, in denen sich die in den Szenarios betrachteten Größen, Zustände und Prozesse unterschiedlich (d. h. auseinander) entwickeln. Sie können für die Entwicklung von Maßnahmen zur Anpassung an den Klimawandel eingesetzt werden. So können Stakeholder alternative Wasserbedarfsszenarien explorativ generieren, die dann zusammen mit Szenarien des Wasserdargebots unter dem Einfluss des Klimawandels für die Identifizierung von Wassermanagementmaßnahmen genutzt werden können.

Während der gemeinsamen Szenarienerstellung werden die Beteiligten ermutigt, ihr Wissen und ihre Problemwahrnehmung über mögliche Zukünfte in einer kreativen Art und Weise zu teilen. Die systematische Exploration der Zukunft erhöht also das (gemeinsame) Systemverständnis und verringert dadurch epistemische Unsicherheit und Uneindeutigkeit. Durch die gemeinsame Entwicklung qualitativer Szenarien wird das Wissen um die Unsicherheit bestimmter Faktoren und die Bedeutung für die Entscheidungsfindung bei den Stakeholdern gestärkt (Amer et al. 2013).

Die flache bis mittlere epistemische Unsicherheit, die bei der Quantifizierung von zukünftigen Gefahren des $\mathrm{KW}$ unter Annahme eines bestimmten Emissionsszenarios auftritt, kann heutzutage recht gut durch die Berechnungsergebnisse von Multi-Modell-Ensembles berücksichtigt werden. Solche Ergebnisse werden 
z. B. im Rahmen der ISIMIP-Initiative (www.isimip.org) erarbeitet und zur Verfügung gestellt. Dabei werden verschiedene Impaktmodelle, z. B. globale hydrologische Modelle, mit dem bias-korrigierten Output einiger globaler Klimamodelle angetrieben. Alle Modellkombinationen (aus Klimamodell und Impaktmodell) zusammen bilden ein Ensemble, sodass bei x Modellkombinationen z. B. x alternative Schätzungen der prozentualen Änderungen der Grundwasserneubildungsrate aufgrund des Klimawandels vorliegen. Diese alternativen Abschätzungen einer KW-Gefahr erlauben nicht nur eine Abschätzung der Gefahr, sondern auch eine Abschätzung der Unsicherheit der Gefahrenabschätzung. Eine Herausforderung ist, die umfangreichen Berechnungsergebnisse von Multi-Modell-Ensembles so darzustellen, dass die angenähert quantifizierbaren Unsicherheiten für die Risikobewertung und die Identifizierung von Managementmaßnahmen optimal berücksichtigt werden können.

Abb. 2 zeigt ein Beispiel für eine neuartige Darstellung der epistemischen Unsicherheit, die durch ein Multi-Modell-Ensemble quantifiziert wurde. Dargestellt ist, wie sich möglicherweise in jeder $0.5^{\circ}$ mal $0.5^{\circ}$ großen Berechnungszelle (55 km mal $55 \mathrm{~km}$ am Äquator) der Durchfluss in Flüssen im Vergleich zum Beginn dieses Jahrhunderts ändern wird, wenn die globale Mitteltemperatur $2{ }^{\circ} \mathrm{C}$ höher sein wird als in vorindustriellen Zeiten. Das Ensemble besteht aus acht Modellkombinationen (MK), wobei zwei globale hydrologische Modelle durch die bias-korrigierten Ergebnisse von vier globalen Klimamodellen angetrieben wurden (Döll et al. 2018). In Abb. 2a ist der Mittelwert der prozentualen Änderungen aller acht MK dargestellt, da im Allgemeinen angenommen wird, dass sich die Fehler der einzelnen MK ausmitteln und daher der Ensemble-Mittelwert die beste Schätzung und wahrscheinlichste Änderung darstellt. Dabei wird die Unsicherheit, die sich über die Variabilität der Ergebnisse der acht MK ausdrückt, dadurch gezeigt, dass der Mittelwert der prozentualen Änderungen nur in den Berechnungszellen in kräftigen Farben dargestellt ist, in denen mindestens sechs der acht MK im Vorzeichen der Änderungen (Zunahme oder Abnahme) übereinstimmen. Die gesamte Spannweite der durch die einzelnen MK berechneten prozentualen Änderungen zeigen Abb. 2b und 2c. Die Karte in Abb. 2b zeigt für jede $0.5^{\circ}$-Zelle das Ergebnis der MK, die für die prozentuale Änderung den kleinsten Absolutwert (unabhängig vom Vorzeichen) aller MK berechnet hat. Da Änderungen egal in welche Richtung problematisch sind, kann dies als bester Fall, d. h. die positivste Schätzung der KW-Gefahr, gesehen werden. Schlimmstenfalls sollten die in Abb. 2c dargestellten prozentualen Änderungen auftreten.

„Eine Einschätzung und Bewertung der größtmöglichen Bandbreite potenzieller Folgen, einschließlich sehr unwahrscheinlicher Folgen mit schwerwiegenden 

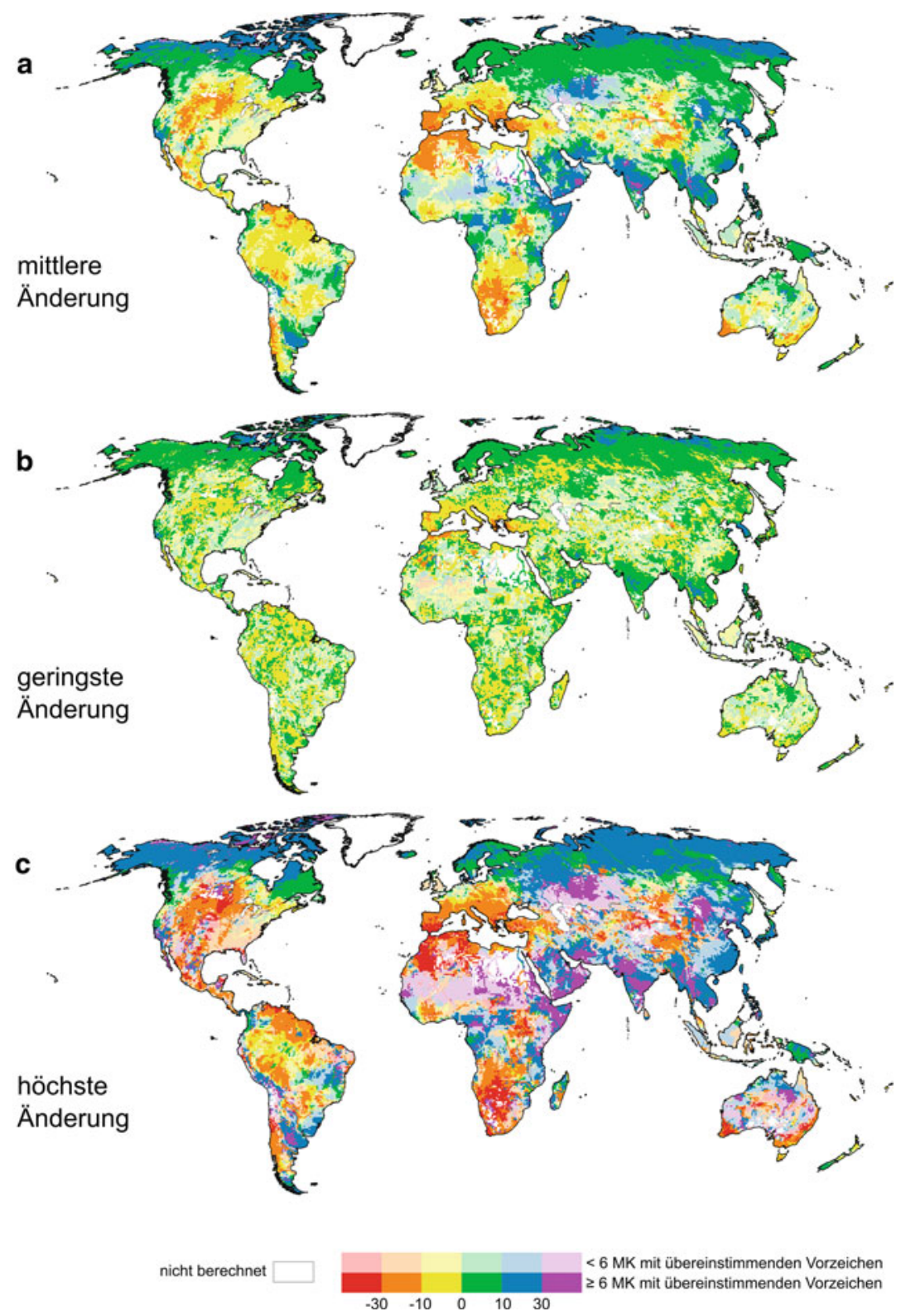
\Abb. 2 Prozentuale Änderung des Durchflusses in Flüssen zwischen dem Beginn des 21. Jahrhunderts und einer Welt, in der die globale Mitteltemperatur um $2{ }^{\circ} \mathrm{C}$ gegenüber der vorindustriellen Zeit erhöht ist. Daten eines Ensembles von acht Modellkombinationen (MK), wobei zwei globale hydrologische Modelle jeweils durch die bias-korrigierten Ergebnisse von vier globalen Klimamodellen angetrieben wurden (Döll et al. 2018). a Arithmetisches Mittel der prozentualen Änderungen aller Modellkombinationen. b „Best case“ mit geringster Änderung des Durchflusses aufgrund des Klimawandels. Dargestellt ist für jede $0,5^{\circ}$-Zelle die prozentuale Änderung, die von der MK mit dem niedrigsten Absolutwert der prozentualen Änderung berechnet wird, unabhängig vom Vorzeichen. c „Worst case“ mit höchster Änderung. In abgeschwächten Farben sind die $0,5^{\circ}$-Zellen dargestellt, bei denen weniger als sechs der acht Modellkombinationen im Vorzeichen der Änderung übereinstimmen

Konsequenzen, ist zentral für das Verständnis der Vor- und Nachteile alternativer Risikomanagementmaßnahmen“ (IPCC 2014, S. 9). Daher sollten nicht nur Ensemblemittelwerte betrachtet werden, sondern auch weniger wahrscheinliche Zukünfte mit großen potentiellen Auswirkungen. Crosbie et al. (2013) produzierten mehrere Karten für den australischen Kontinent, auf denen die zukünftigen Änderungen der erneuerbaren Grundwasserressourcen, die mit unterschiedlichen Wahrscheinlichkeiten nicht überschritten werden, dargestellt sind. Solche Karten unterstützen, ebenso wie die in Abb. 2 gezeigten, Stakeholder mit unterschiedlicher Risikobereitschaft bei der Entscheidungsfindung. So würden Stakeholder mit sehr hoher Risikobereitschaft eine Anpassung an die minimalen prognostizierten Veränderungen von Abb. $2 b$ für ausreichend halten, während Stakeholder mit einem hohen Sicherheitsbedarf Anpassungsmaßnahmen (z. B. zur Verringerung der Wassernutzung) bevorzugen würden, die auch bei den starken Änderungen des Durchflusses, wie sie in Abb. 2c gezeigt werden, zu einer guten Wasserressourcensituation führen.

\section{Schlussfolgerungen}

Aufgrund der Komplexität des Erdsystems (einschließlich der menschlichen Gesellschaft) gibt es große Unsicherheiten bei der Abschätzung potenzieller zukünftiger Klimaänderungen und der sich daraus ergebenden Gefahren und Risiken. Bekanntermaßen führt eine ungenügende Berücksichtigung von Unsicherheiten zu suboptimalen Entscheidungen (Bastin et al. 2013). Dabei sind Unsicherheiten für die Entwicklung von Anpassungsmaßnahmen bedeutender als für die Entwicklung von Klimaschutzmaßnahmen. Partizipative transdisziplinäre Prozesse mit Stakeholdern und Wissenschaftler*innen sind am besten 
für die Bewertung von Klimawandelrisiken und die Entwicklung von Risikomanagementmaßnahmen auf lokaler bis nationaler Skala geeignet. In solchen PRMP sollten die vielfältigen relevanten Unsicherheiten explizit und in geeigneter Form berücksichtigt und diskutiert werden. Dazu können Methoden, wie sie in Kap. 3 und detaillierter in Döll und Romero-Lankao (2017) beschrieben sind, angewendet werden. Die in Kap. 2 vorgestellte Unsicherheitsklassifikation erleichtert die Erfassung und Strukturierung der im spezifischen Kontext relevanten Unsicherheiten.

Von Bedeutung für den Umgang mit Unsicherheiten beim Management von KW-Risiken ist auch, wie Unsicherheiten kommuniziert werden, z. B. von Wissenschaftler*innen in wissenschaftlichen Publikationen und Journalist*innen in Massenmedien. Nach einer Analyse von Abstracts wissenschaftlicher Publikationen forderten Guillaume et al. (2017) ihre Kolleg*innen auf, bewusster und expliziter als bislang die Unsicherheit der präsentierten Forschungsergebnisse sprachlich darzustellen. Die Linguistinnen Simmerling und Janich (2016) beschrieben am Beispiel eines deutschsprachigen Zeitungsartikels zu Geoengineering die Komplexität der „Unsicherheitssprache“ von Journalist*innen, mit ihren vielfältigen linguistischen Mitteln und rhetorischen Funktionen. Um das Management der KW-Risiken zu verbessern, empfehle ich, verstärkt zur Charakterisierung und Kommunikation verschiedenartiger Unsicherheiten sowie zur bestmöglichen Berücksichtigung von Unsicherheiten bei Entscheidungen zu forschen.

Danksagung Ich danke Hans-Peter Rulhof-Döll für die Erstellung von Abb. 1 und Tim Trautmann für die Erstellung von Abb. 2.

\section{Literatur}

Amer, M., T. U. Daim, und A. Jetter. 2013. A review of scenario planning. Futures 46:23-40. https://doi.org/10.1016/j.futures.2012.10.003.

Ascough II, J. C., H. R. Maier, J. K. Ravalico, und M. W. Strudley. 2008. Future research challenges for incorporation of uncertainty in environmental and ecological decision-making. Ecological Modelling 219 (3):383-399. https://doi.org/10.1016/j.ecolmodel.2008.07.015.

Bastin, L., D. Cornford, R. Jones, G. B. M. Heuvelink, E. Pebesma, C. Stasch, S. Nativi, P. Mazzetti, und M. Williams. 2013. Managing uncertainty in integrated environmental modelling: The UncertWeb framework. Environmental Modelling \& Software 39:116134. https://doi.org/10.1016/j.envsoft.2012.02.008.

Bijlsma, R. M., P. W. G. Bots, H. A. Wolters, und A. Y. Hoekstra. 2011. An empirical analysis of stakeholders' influence on policy development: The role of uncertainty handling. Ecology and Society 16:51. http://www.ecologyandsociety.org/vol16/iss1/art51/. 
Carey, J. M., und M. A. Burgman. 2008. Linguistic uncertainty in qualitative risk analysis and how to minimize it. Annals of the New York Academy of Sciences 1128:13-17. https:// doi.org/10.1196/annals.1399.003

Crosbie, R. S., et al. 2013. An assessment of the climate change impacts on groundwater recharge at a continental scale using a probabilistic approach with an ensemble of GCMs. Climatic Change 117 (1-2):41-53. https://doi.org/10.1007/s10584-012-0558-6.

Döll, P., T. Trautmann, D. Gerten, H. Müller, S. Ostberg, F. Saaed, und C.-F. Schleussner. 2018. Risks for the global freshwater system at $1.5^{\circ} \mathrm{C}$ and $2{ }^{\circ} \mathrm{C}$ global warming. Environmental Research Letters 13:044038. https://doi.org/10.1088/1748-9326/aab7.

Döll, P., und P. Romero-Lankao. 2017. How to embrace uncertainty in participatory climate change risk management - A roadmap. Earths Future 5:18-36. https://doi.org/10.1002/ 2016 EF000411.

Döll, P., B. Jiménez-Cisneros, T. Oki, N. Arnell, C. Benito, G. Cogley, T. Jiang, Z. W. Kundzewicz, S. Mwakalila, und A. Nishijima. 2015. Integrating risks of climate change into water management. Hydrological Sciences Journal 60 (1):3-14. https://doi.org/10.1080/ 02626667.2014.967250.

Döll, C., P. Döll, und P. Bots. 2013. Semi-quantitative actor-based modeling as a tool to assess the drivers of change and physical variables in participatory integrated assessments. Environmental Modelling \& Software 46:21-32. https://doi.org/10.1016/j.envsoft.2013. 01.016.

Döll, P. 2009. Vulnerability to the impact of climate change on renewable groundwater resources: A global-scale assessment. Environmental Research Letters 4:036006. https:// doi.org/10.1088/1748-9326/4/3/035006.

Düspohl, M., und P. Döll. 2016. Causal networks and scenarios: Participatory strategy development for promoting renewable electricity generation. Journal of Cleaner Production 121:218-230. https://doi.org/10.1016/j.jclepro.2015.09.117.

Guillaume, J. H. A., C. Helgeson, S. Elsawah, A. J. Jakeman, und M. Kummu. 2017. Toward best practice framing of uncertainty in scientific publications: A review of Water Resources Research abstracts. Water Resources Research 53:6744-6762. https://doi.org/10.1002/201 7WR020609.

IPCC. 2014. Zusammenfassung für politische Entscheidungsträger In Klimaänderung 2014: Folgen, Anpassung und Verwundbarkeit. Beitrag der Arbeitsgruppe II zum Fünften Sachstandsbericht des Zwischenstaatlichen Ausschusses für Klimaänderungen (IPCC), Hrsg. Christopher B. Field et al., 1-32. Cambridge und New York: Cambridge University Press. Deutsche Übersetzung durch Deutsche IPCC-Koordinierungsstelle, Österreichisches Umweltbundesamt, ProClim, Bonn/Wien/Bern.

Kwakkel, J. H., W. E. Walker, und V. A. W. J. Marchau. 2010. Classifying and communicating uncertainties in model-based policy analysis. International Journal of Technology, Policy and Management 10 (4):299-315. https://doi.org/10.1504/ijtpm.2010.036918.

Lang, D. J., A. Wiek, M. Bergmann, M. Stauffacher, P. Martens, P. Moll, M. Swilling, und C. J. Thomas. 2012. Transdisciplinary research in sustainability science: Practice, principles, and challenges. Sustainability Science 7 (1):25-43. https://doi.org/10.1007/s11625-0110149-x.

Mastrandrea, M., K. Mach, G.-K. Plattner, O. Edenhofer, T. Stocker, C. Field, K. Ebi, und P. Matschoss. 2011. The IPCC AR5 guidance note on consistent treatment of uncertainties: A 
common approach across the working groups. Climatic Change 108 (4):675-691. https:// doi.org/10.1007/s10584-011-0178-6.

Milly, P. C. D., J. Betancourt, M. Falkenmark, R. M. Hirsch, Z. W. Kundzewicz, D. P. Lettenmeier, und R. J. Stouffer. 2008. Stationarity is dead: Whither water management? Science 389:573-574. https://doi.org/10.1126/science.1151915.

Mimura, N., R. S. Pulwarty, D. M. Duc, I. Elshinnawy, M. H. Redsteer, H. Q. Huang, J. N. Nkem, und R. A. Sanchez Rodriguez. 2014. Adaptation planning and implementation. In Climate change 2014: Impacts, adaptation, and vulnerability. Part A: Global and sectoral aspects contribution of working Group II to the fifth assessment report of the intergovernmental panel on climate change, 869-898, Hrsg. Christopher B. Field, et al., 1-32. Cambridge und New York: Cambridge University Press.

Renn, O., A. Klinke, und M. van Asselt. 2011. Coping with complexity, uncertainty and ambiguity in risk governance: A synthesis. Ambio 40 (2):231-246. https://doi.org/10. 1007/s13280-010-0134-0.

Renn, O. 2008. Risk Governance: Coping With Uncertainty in a Complex World. London: Routledge.

Scholz, R., und G. Steiner. 2015. The real type and ideal type of transdisciplinary processes: Part II-what constraints and obstacles do we meet in practice? Sustainability Science 10 (4):653-671. https://doi.org/10.1007/s11625-015-0327-3.

Simmerling, A., und N. Janich. 2016. Rhetorical functions of a 'language of uncertainty' in the mass media. Public Understanding of Science 25 (8):961-975. https://doi.org/10. 1177/0963662515606681.

Titz, A., und P. Döll. 2009. Actor modelling and its contribution to the development of integrative strategies for management of pharmaceuticals in drinking water. Social Science \& Medicine 68 (4):672-681. https://doi.org/10.1016/j.socscimed.2008.11.031.

Van den Hoek, R. E., M. Brugnach, J. P. M. Mulder, und A. Y. Hoekstra. 2014. Analysing the cascades of uncertainty in flood defence projects: How "not knowing enough" is related to "knowing differently". Global Environmental Change 24:373-388. https://doi.org/10. 1016/j.gloenvcha.2013.11.008.

Van Notten, S. 2006. Scenario development: A typology of approaches. In Think Scenarios, Rethink Education, 69-92. Paris: OECD Publishing. https://doi.org/10.1787/978926 4023642-en. https://www.oecd-ilibrary.org/education/think-scenarios-rethink-education/ scenario-development_9789264023642-6-en.

Walker, W. E., P. Harremoes, J. Rotmans, J. P. van der Sluijs, M. B. A. van Asselt, P. Janssen, und M. P. Krayer von Krauss. 2003. Defining uncertainty: A conceptual basis for uncertainty management in model-based decision support. Integrated Assessment 4 (1):5-17. https://doi.org/10.1076/iaij.4.1.5.16466

Döll, Petra, Prof. Dr., Professur für Hydrologie am Fachbereich Geowissenschaften/Geographie der Goethe-Universität Frankfurt am Main, assoziiert mit dem Senckenberg Leibniz Biodiversität- und Klimaforschungszentrum (SBiK-F), Frankfurt am Main. http://www.uni-frankfurt.de/45217668/dl p.doell@em.uni-frankfurt.de. 
Open Access Dieses Kapitel wird unter der Creative Commons Namensnennung 4.0 International Lizenz (http://creativecommons.org/licenses/by/4.0/deed.de) veröffentlicht, welche die Nutzung, Vervielfältigung, Bearbeitung, Verbreitung und Wiedergabe in jeglichem Medium und Format erlaubt, sofern Sie den/die ursprünglichen Autor(en) und die Quelle ordnungsgemäß nennen, einen Link zur Creative Commons Lizenz beifügen und angeben, ob Änderungen vorgenommen wurden.

Die in diesem Kapitel enthaltenen Bilder und sonstiges Drittmaterial unterliegen ebenfalls der genannten Creative Commons Lizenz, sofern sich aus der Abbildungslegende nichts anderes ergibt. Sofern das betreffende Material nicht unter der genannten Creative Commons Lizenz steht und die betreffende Handlung nicht nach gesetzlichen Vorschriften erlaubt ist, ist für die oben aufgeführten Weiterverwendungen des Materials die Einwilligung des jeweiligen Rechteinhabers einzuholen.

(c) (1) 